\title{
Apendicitis aguda en el niño
}

\author{
Publicado en Revista Chilena de Pediatría 1932
}

Autor: ROBERTO KOHAN ${ }^{\dagger}$
Médico Agregado del Hospital Arriarán. Médico-Jefe del Policlínico Israelita.

Comentario: ALEJANDRO ZAVALA B. ${ }^{1}$, BEATRIZ ZAVALA P. ${ }^{2}$, FRANCISCO VERA O. ${ }^{2}$, LUISA SCHONHAUT B ${ }^{3}$

1. Profesor Asistente, Departamento Cirugía, Sección Cirugía Infantil, Pontificia Universidad Católica de Chile.

2. Internos $7^{a}$ año Medicina Universidad del Desarrollo.

3. Profesor. Pediatra, Departamento de Pediatría. Facultad de Medicina Clínica Alemana - Universidad del Desarrollo.

Key words: Appendicitis, Children, pediatric history.

Palabras clave: Apendicitis, Niño, historia de la pediatría.

\section{Introducción}

La apendicitis aguda es la patología abdominal quirúrgica de urgencia más frecuente en el niño ${ }^{1}$. Desde 1886 en que Fitz ${ }^{2,3}$ acodó el nombre de apendicitis, describió su fisiopatología y propuso la apendicectomía, quedaron atrás los diagnósticos de tiflitis, peritiflitis y paratiflitis.

Si bien su morbilidad y mortalidad han disminuido significativamente, aún en nuestros tiempos de evidencia médica bien fundamentada, existen controversias y resistencia frente a la incorporación de nuevas propuestas diagnósticas y terapéuticas: "Es un modelo de vanidades humanas en tiempos de poca base cientifica y mucha interpretación observativa" (Williams 1983)"; "La historia de la apendicitis presenta ejemplos de gran resistencia al cambio de conceptos, de tempranas y brillan- tes observaciones no aceptadas, y de apoyos emocionales para posturas no defendibles. Del momento adecuado y del desarrollo de una muy satisfactoria solución" (Williams 1983)5.

La clínica, en la actualidad, tiene en muchos centros un valor secundario frente a los estudios de imágenes. Una clara explicación para ello es el intento de disminuir los errores diagnósticos y las complicaciones; y otra justificación no tan clara, pero entendible, es la validación del acto médico en tiempos de desconfianzas. La toma de decisiones en medicina es un proceso complejo, que se basa en la interacción del médico, el paciente y el entorno, donde la clínica se complementa y le da matices a los resultados de laboratorio e imágenes. Todo este proceso es interpretativo, siendo fundamental la comunicación entre el radiólogo, el pediatra y el cirujano en las conclusiones obtenidas.

Rescatamos y resumimos un trabajo de Apendicitis Aguda publicado por el Dr. Roberto Kohan en la Revista Chilena de Pediatría de $1932^{6}$. En el artículo se relatan 4 casos clínicos muy descriptivos, analizamos uno para observar la secuencia diagnóstica y terapéutica de

Trabajo recibido el 1 de julio de 2012, aceptado para publicación el 22 de agosto de 2012.

Correspondencia a:

Alejandro Zavala B.

E-mail: azavala@vtr.net 
esa época donde era la clínica, y no las imágenes, la base del diagnóstico. Queremos destacar que, a pesar que se han agregado elementos tecnológicos al estudio de la apendicitis, su semiología básica no ha sufrido variaciones a lo largo de la historia de la pediatría

\section{Resumen Artículo}

"Los síntomas deben evaluarse y no contarse" PAYR.

La apendicitis aguda del niño no tiene la evolución clínica de la del adulto. Los accidentes se suceden con mayor rapidez y adquieren mayor gravedad. Las condiciones de infección son las mismas que en el adulto; debe considerarse entonces el terreno como el factor que da a la infección un giro clínico diferente.

Ombredanne y otros autores han establecido que el niño, en el curso de la apendicitis, con cierta frecuencia, tiene reacciones orgánicas diferentes a las del adulto.

En verdad se sabe que pocas afecciones proporcionan la oportunidad de que se cometan tan variados errores de diagnóstico como las enfermedades del apéndice. Ello se debe a la situación muy variable de este órgano y sus relaciones anatómicas con las partes vecinas, al distinto grado de las alteraciones anatomopatológicas y a la irregularidad, a la inconstancia y a la vaguedad con que muchas veces se presentan algunos de sus síntomas (Schwalbe).

\section{Etiología y patología}

¿Cuál es la causa que hace estallar la crisis aguda? ¿Es la exaltación de la virulencia de las colonias microbianas que han infectado los folículos linfoideos del apéndice o es una infección nueva sobreagregada? El hecho importante es que la infección apendicular se localiza sobre los folículos linfáticos, tan numerosos a nivel del apéndice. Pero, al lado de la infección existen otros factores que favorecen el estallido del proceso agudo apendicular. Ombredanne, al referirse a los cálculos estercoráceos, masas ovoideas de materias fecales endurecidas, considera que estos cálculos no son la causa de la apendicitis aguda; ellos pa- recen más bien ser el efecto de la evolución de las lesiones de apendicitis crónicas; sin embargo, su efecto parece innegable en el momento del estallido de los accidentes.

Lanz no da importancia a la existencia del oxiuro en el apéndice y dice que "si es verdad que los oxiuros se encuentran muchas veces en el interior de los apéndices enfermos, no es menos cierto que se les encuentra en los apéndices sanos con igual frecuencia", Metchnioff, a su vez, les asigna gran significación: "el oxiuro actuaría por acción traumática, de expoliación irritativa e inflamatoria y finalmente infecciosa".

\section{Clínica}

Raquel B, 8 años, N. Carátula 433, 6-11-31, H. Arriarán.

Antecedentes, sin importancia.

Enfermedad actual: Ayer (5-11-31) en la mañana, principió a quejarse de dolores peri umbilicales que, en la tarde, se localizaron en la fosa iliaca derecha. Como el cuadro se agravara, la madre le dio un purgante de aceite de ricino (una cucharada de postre), y, como no hiciera efecto, a las dos horas le hizo un lavado con aceite de olivas: al poco rato tuvo una deposición normal, pero los dolores abdominales siguieron con igual intensidad. La temperatura, que en un principio era apenas subfebril, llegó a $38.5^{\circ}$ en la mañana de hoy. No han tenido vómitos.

Acudió a la A.P. de donde es enviada con el diagnóstico de apendicitis aguda.

Examen: Estado general bueno. Psiquis despejado, Facies febril. Sin disnea ni aleteo nasal. Nada pulmonar ni faríngeo. Dolor a la palpación profunda en la fosa iliaca derecha. Más en el punto de Mc Burney. Sin hiperestesia cutánea ni defensa muscular. Signos del Psoas y de Blumberg positivos. 110.

Temperatura axilar $37.6^{\circ}$; rectal $39^{\circ}$; pulso

Tacto rectal: Sensible el fondo de saco derecho.

Hemograma:

\begin{tabular}{|c|c|c|c|c|c|c|c|}
\hline Bas & Eos & Miel & Jov & Bac & Segm & Linf & Mono \\
\hline & & & 2 & 11 & 50 & 37 & \\
\hline
\end{tabular}


Tomando en consideración el dolor marcado que existía en el punto de Mc Burney, los signos del psoas y de Blumberg positivos, el tacto rectal doloroso, la diferencia de más de un grado entre la temperatura axilar y la rectal, y el hemograma, se pensó en una apendicitis aguda, y con tal diagnóstico se intervino.

Intervención: Apéndice engrosada, muy largo, de color concho-vino en su extremidad distal. Grueso ganglio en la base del apéndice. Apendicectomía.

Evolución: sin complicaciones.

$$
\text { 11-11-31 }
$$

\begin{tabular}{|ccccccc|}
\hline Bas Eos Miel & Jov & Bac & Segm & Linf & Mono \\
1 & & 1 & 8 & 60 & 28 & 2 \\
\hline Gl Blancos: 8.000 & & & & & \\
\hline
\end{tabular}

Es dada de alta en buenas condiciones, 14 días después de su hospitalización.

Diagnóstico: Apendicitis aguda.

En la sintomatología de la apendicitis consideramos, en primer término, el período en que aparecen signos de la afección. Dice Murphy, al referirse a la apendicitis aguda del adulto, que los síntomas se desarrollan en el siguiente orden: Dolor, vómitos, sensibilidad iliaca, fiebre, leucocitosis. Y declara Murphy, todavía: "los síntomas aparecen siempre, sin excepción, en el orden citado, y cuando ese orden varía, pongo en duda el diagnóstico. Sin embargo, en el niño no se puede aceptar el mismo temperamento, porque el orden indicado arriba, varia con cierta frecuencia. En algunos casos el vómito falta en absoluto. Sólo por excepción comienza la apendicitis con temperaturas elevadas. Finalmente a veces inicia en el orden indicado por Murphy.

Al estudiar los antecedentes del enfermo debemos saber si el niño en alguna ocasión ha eliminado o no vermes intestinales, porque este dato, muchas veces, puede servirnos para precisar un diagnóstico.

En la crisis apendicular, simple, benigna, la enfermedad comienza bruscamente, sin causa aparente alguna, con un dolor vivo en el abdomen. Esta brusquedad es tal que hasta se puede precisar la hora exacta del comienzo de los accidentes. Otras veces, no frecuentes por cierto, el proceso comienza en forma insidiosa.

El dolor espontáneo a menudo se localiza a nivel del epigastrio; al cabo de pocas horas se localiza en la fosa iliaca derecha. Otras veces el dolor abdominal es difuso, sin tendencia a la localización, sino después de muchas horas. A su vez la Clínica nos enseña que el niño, con regular frecuencia, refiere su dolor únicamente a la fosa iliaca derecha.

En el niño es frecuente que el dolor se haga en forma continua.

Mientras en el adulto se describen una serie de puntos dolorosos, que serían propios de la apendicitis (puntos de Mc Burney, de Morris, de Lanz, de Lentzmann, de Munro, de Clado, de Widal, etc) en el niño es bastante difícil precisar un punto doloroso, por la razón que el dolor se nota, en forma más o menos igual, en toda una zona. Cuanto más joven es el enfermo, tanto más difícil es encontrar un punto doloroso. En el niño, por encima de los ocho años, el dolor tiende a presentar todos los caracteres del dolor de las apendicitis del adulto.

Estado general: En el 60\% de los casos, el estado general permanece bueno. Y el hecho que el niño se encuentre aparentemente en buenas condiciones, tranquilo, llegando al Hospital muchas veces por sus pies, debe considerarse con mucha cautela, porque puede inducirse a los mayores errores de diagnóstico.

En un momento dado, sin causa aparente alguna, el estado general decae intensamente. La mirada empañada de los ojos, la nariz afilada y las mejillas frías, nos demuestran que el niño está en un estado de intoxicación grave y que la vida del enfermo está en inminente peligro.

Lengua: Según nuestras estadísticas, en el $80 \%$ de los casos, se presenta saburral; sólo por excepción se encuentra limpia y húmeda.

Temperatura y pulso: La temperatura sube lentamente, hasta que, horas después, alcanza a $38^{\circ}$. En algunos casos falta la reacción térmica.

Dice Schwalbe que "la diferencia sobre un grado entre la temperatura axilar y rectal (signo de Madelung) si no está por completo desprovisto de valor, es de escasa importancia en la práctica". Desde luego el signo de Madelung no es frecuente en las afecciones apendiculares agudas, por lo menos en de los niños, $\mathrm{y}$, aun más, suele hallarse, como hemos teni- 
do ocasión de comprobarlo en cierto número de afecciones que nada tenían que ver con la apendicitis aguda.

Hemos dicho que al comienzo de un ataque apendicular agudo, la temperatura es ordinariamente normal, pero que, al cabo de pocas horas, se eleva a $37,5-38^{\circ}$. Si en algún enfermo, con dolor abdominal, al iniciarse la enfermedad, se encuentra una temperatura de $40-41^{\circ}$, es muy probable que el tórax o el riñón sean el asiento de la afección. Y lo decimos así, porque es absolutamente raro que la apendicitis aguda se inicie con temperaturas elevadas.

En la apendicitis aguda casi siempre hay taquicardia; pero, no olvidemos que el pulso y la temperatura son elementos engañadores. Hay una serie de condiciones que hacen que las reacciones del organismo sobre una infección sean atípicas. Sin embargo, tiene importancia la divergencia entras las curvas de temperatura y el pulso (singo de Jalaguier). Una temperatura apenas sub-febril y un pulso de 150 deben hacernos pensar que existe una causa que necesita, por parte del médico, una solución diagnóstica urgente. (Ombredanne).

Respiraciones: En general el número de respiraciones es normal o apenas aumentado; si es el doble de lo normal, al comienzo de la enfermedad, es muy probable que la lesión causal tenga un origen torácico.

Defensa muscular: en la apendicitis aguda es la defensa muscular un signo de vital importancia. Mientras el niño grita, no hay que pensar en determinar la tensión muscular aumentada.

Es preciso distraer al enfermo y esperar, con mucha paciencia, un momento en que esté quieto, para poder percibir bien la tensión refleja de la pared abdominal.

La defensa muscular falta cuando: a) ha pasado cierto tiempo de la iniciación del proceso; b) hay un empiema del apéndice; c) el colon ascendente se encuentra en un sitio profundo de la excavación pélvica; d) apéndice posterior (Payr).

Los signos de Blumberg, Jacob, Sherren, del obturador, del psoas, etc., se podrán encontrar con relativa frecuencia en los niños mayores pero en el lactante y en el niño muy pequeño sería inútil buscarlos, pues al paciente no nos diría que sensaciones sufre al hacer tal o cual movimiento o al tocar en una u otra zona del abdomen.

Orina: Los dolores y molestias al orinar constituyen un síntoma importante de apendicitis siempre que la orina sea normal (Feer).

Deposiciones: Por lo general hay constipación; raras veces, diarreas. Cuando en un lactante se desarrolla un cuadro apendicular agudo con diarrea y temperaturas elevadas, se pensará siempre en una peritonitis neumocócica (Davioud y Michaut).

Tacto rectal: Es un valioso medio de diagnóstico que, por desgracia, es olvidado muchas veces por el clínico. Permite reconocer a veces una apendicitis o un absceso apendicular cuando el examen abdominal es todavía completamente negativo. Es por esto que el profesor Sierra nos repetía constantemente que "es mucho más importante introducir el dedo en el recto, que colocar el termómetro en la axila".

\section{Diagnóstico}

1.- Peritonitis apendicular; 2.- Peritonitis tuberculosa; 3.- Helmintiasis; 4.- Neumonía y Gripe; 6.- Pielitis; 7.- Peritonitis neumocócica; 8.- Fiebre tifoidea; 9.- Invaginación Intestinal; 10.- No se ha de tener en cuenta casi ninguna de las afecciones genitales de la mujer (excluyendo la peritonitis gonocócica) y también, por otra parte, porque puede decirse que falta la posibilidad de la úlcera gástrica o intestinal, del cáncer gástrico, así como la de un cólico hepático o nefrítico o una enfermedad del páncreas.

\section{Discusión del Artículo Dr. Roberto Kohan ${ }^{\dagger}$}

Kohan en su artículo se basa en la experiencia de 185 casos de apendicitis aguda (115 casos, Hospital del Río; 70 casos, H. Arriarán) operados entre 1925 y mediados de 1931, con una tasa de mortalidad de $1,65 \%$. La mayor frecuencia de cirugías fue en niños mayores de 10 años de edad, "Por debajo de los cinco años se han presentado sólo tres casos de apendicitis aguda, sin complicaciones."(Kohan 1932).

Es claro que tanto el adulto como el niño comparten la fisiopatología de la apendicitis, 
es el "terreno lo que le da un giro diferente". Según refiriera Diaz Burdeau 16 años después, en 1948 "el aumento de las apendicitis en niños menores de 3 años es sólo aparente, pues este cuadro se negaba por desconocimiento de su existencia"7. En los más pequeños los síntomas y signos son vagos e inespecíficos, y además difíciles de precisar, por este motivo frecuentemente se diagnostican en etapa de complicación ${ }^{8,9}$.

Un niño que se siente mal, está asustado, con muchas personas desconocidas, en un lugar no grato como son las urgencias y con personal médico habitualmente presionado por la demanda asistencial, no siempre va a cooperar para lograr identificar la sensibilidad abdominal. Esto obliga a dominar técnicas de acercamiento y distracción que permitan en corto tiempo poder examinar en forma adecuada al menos a un grupo importante de estos pacientes. Si bien los estudios de imágenes complementan una clínica dudosa, no nos aportan certeza absoluta ${ }^{10}$, por lo que la observación acuciosa acompañada de la sospecha clínica son los ejes del diagnóstico oportuno.

El tacto rectal es un procedimiento que cada día se realiza menos por lo traumático que resulta y por la poca información adicional que otorga en relación a una buena ecografía $^{11}$. En casos dudosos, sin exámenes de imagen, o con un absceso pélvico es de gran ayuda, previa preparación del niño o niña. En el texto se describe que en esa época también tendía a olvidarse, a pesar que "el profesor Sierra nos repetía constantemente que "es mucho más importante introducir el dedo en el recto, que colocar el termómetro en la axila". Hace casi 40 años, en 1976, Dolz publicaba "el tacto rectal se efectuó en 1 de cada 5 pacientes y fue ayuda diagnostica en el $84 \%$, por lo que no debe omitirse" 12 . La omisión de este procedimiento, en tiempos en que no contábamos con imágenes, probablemente sólo refleja lo molesto y difícil que es realizarlo en pediatría.

La evolución de los diagnósticos diferenciales de la Apendicitis Aguda son reflejo de los avances de la salud pública y del saneamiento ambiental que ha experimentado el país. Es así como entre los principales diagnósticos diferenciales, hace 75 años se encon- traban la peritonitis tuberculosa, la fiebre tifoidea y las parasitosis, enfermedades que hoy en día son de excepción. Hace 50 años los Drs. Camacho y Vera destacaban la presencia de ascaridiasis en los niños fallecidos por apendicitis aguda, por lo que consideraban "imperativo la busqueda y tratamiento oportuno de estos vermes, especialmente en les enfermos menores de 4 años"13. En las últimas 3 décadas la portación de parásitos patógenos intestinales descendió del $9,8 \%$ a $2,5 \%{ }^{14}$ y la TBC, que en los años 30 tenía una mortalidad de 253,4 por 100000 habitantes, en el 2002 descendió a 2 por $100000 \mathrm{hab}^{15}$. Dos de los diagnósticos diferenciales de la apendicitis aguda, frecuentes hoy y no mencionados en este artículo, son la adenitis mesentérica y la constipación ${ }^{16,17}$, esta última también relacionada con los cambios de hábitos en nuestra población ${ }^{18}$.

Los embarazos en adolescentes eran y siguen siendo un problema relevante de salud pública; al respecto, debemos mencionar que se estima que un $20 \%$ de las mujeres inician la actividad sexual entre los 12-18 años y del total de recién nacidos vivos, el año 2009 en Chile, $16 \%$ fueron hijos de madres adolescentes ${ }^{19,20}$ $\mathrm{y}$, en aquellos años, además las enfermedades de transmisión sexual en adolescentes eran frecuentes $^{21}$. Es por esto que llama la atención el comentario del Dr. Kohan respecto a que "no se ha de tener en cuenta casi ninguna de las afecciones genitales de la mujer (excluyendo la peritonitis gonocócica)". Dado que no considerar las afecciones de los genitales femeninos puede llevarnos a cometer errores diagnósticos, se recomienda pedir una ecografía abdomino-pelviana en toda niña, especialmente si ha comenzado a tener actividad sexual. "En verdad se sabe que pocas afecciones proporcionan la oportunidad de que se cometan tan variados errores de diagnóstico como las enfermedades del apéndice"(Kohan 1932). Díaz Bordeu publicó la experiencia de 1,981 niños operados con diagnóstico de Apendicitis en el Hospital de Niños Roberto del Río, entre los años 1940 y 1946, reportando una tasa de peritonitis de $9,3 \%$ y $1,1 \%$ de error diagnóstico " $y$ son ellos los que nos plantean uno de los capitulos más difíciles del tema, cual es el diagnostico diferencial" "T. Trece años después, los Drs. 
Camacho y Vera analizaron la casuística de 1.821 niños operados de apendicitis entre los años 1942 y 1959 inclusive, en el Servicio de Urgencia del Hospital Manuel Arriarán, destacando "el evidente descenso de la incidencia de la complicación peritoneal, que del 27,5\% registrado en el sexenio 1942-1947 ha disminuido a 19,2\% en los últimos seis años"13. El año 1976 los Drs. Dolz y García encontraron una tasa de apéndices necrosados o perforados de $35 \%$ (66\% en menores de 5 años) y $10 \%$ de apéndices sanos, de un total del 250 niños operados de apendicitis durante un período de 18 meses en el Hospital Luis Calvo Mackenna ${ }^{12}$.

Esta información erróneamente podría ser interpretada como un aumento de las complicaciones apendiculares a lo largo de la historia de la pediatría. No obstante, coincidiendo con las estadísticas de Dolz, en nuestros días se acepta hasta un $17 \%$ de apendicectomías en blanco y 20 a $70 \%$ de apéndices complicados, dependiendo de la serie y edades de los pacientes incluidos. Es casi una regla a menor número de apéndices complicados mayor número de apendicectomías en blanco y viceversa, independiente de los estudios de apoyo ${ }^{1,8,10,12,22}$. Las excelentes cifras reportadas por Kohan en los años 30 y Díaz Bordeu en los 40 podrían explicarse por un tema de registro, probablemente varias de las apendicitis complicadas no se diagnosticaban y tampoco se realizaba la histología de rutina.

Kohan planteaba la existencia de dos formas de apendicitis, una destructiva y otra no destructiva, pero no queda claro en el artículo esta diferenciación "Con cierta frecuencia se confunde la forma destructiva de la enfermedad (perforación-gangrena) con la forma no destructiva (apendicitis simple) y vice-versa". Hoy se considera un continuo con distintas etapas de un mismo proceso patológico ${ }^{23}$. En la práctica diaria llaman la atención algunos pacientes con historias muy cortas y con etapas muy avanzadas de la enfermedad apendicular $\mathrm{y}$, por el contrario, historias de varios días con etapas iniciales de apendicitis aguda. Esto hace reflexionar que puede haber en ambos extremos de la distribución normal, formas diferentes de la enfermedad en cuanto a su evolución, probablemente secundario a la forma como se establece la obstrucción del apéndice, a factores de virulencia de las bacterias involucradas o a factores histológicos inmunitarios propios del apéndice ${ }^{24}$.

En los años 30-40 era largo tiempo de evolución intrahospitalario de los niños operados, 14 días, para lo que impresiona como una apendicitis aguda inicial, comparado con estadías de 1 a 3 días en la actualidad. En este análisis hay que pensar que en 1945 comenzaron los primeros usos de la Penicilina en las peritonitis apendiculares como describe el Dr. Rene Artigas Nambrard en el capítulo "La penicilina en paracaídas" de su libro "Sucesos en la vida de un cirujano"25. Según los Drs. Camacho y Vera, a partir del año 1950 se produjo una disminución de un 50\% en la mortalidad producida por apendicitis aguda, "Esto no es sólo consecuencia del advenimiento de los antibióticos, pues se venía observando desde antes de su empleo, sino de un conjunto de medidas entre las que se destacan pre y postoperatorios mejor controlados y superación de las técnicas anestésicas y quirúrgicas" ${ }^{13}$. Un gran cambio en resultados en el manejo de la apendicitis aguda en los 80 fue la incorporación del metronidazol en reemplazo de la penicilina.

Han pasado 126 años desde que Fitz estableció el diagnóstico, la histopatología y definió el tratamiento de la apendicitis aguda y casi 80 años desde la publicación del Dr. Kohan, que comentamos en el presente artículo. A pesar de todos los avances tecnológicos en diagnóstico y tratamiento, la apendicitis aguda sigue siendo un desafío. Sólo el 2011 Pubmed registró 653 artículos bajo el título de apendicitis, lo que indica su relevancia. Hoy se discute la elección del tipo de imagen a solicitar ${ }^{26-28}$, el abordaje si laparoscópico o clásico ${ }^{29-31}$, si se toman cultivos ${ }^{32}$, que esquema antibiótico a usar $^{33,34}$, por cuanto tiempo ${ }^{35}$, y si la apendicitis es una urgencia ${ }^{36}$.

La aparición de nuevos exámenes como la Resonancia Magnética para la apendicitis agu$\mathrm{da}^{37}$ y la incorporación de los equipos 3 Tesla, el riboleucograma con niveles de citoquina ${ }^{38} \mathrm{o}$ nuevas tecnologías por aparecer, traerán consigo nuevas preguntas de costo beneficio y de resistencia al cambio. Esto, sin embargo, no cambiará o mejorará la interacción y comu- 
nicación directa entre el cirujano, radiólogo y pediatra en la toma de decisiones, el compromiso de la relación médico-paciente, la historia clínica y el examen físico dedicado, la reevaluación periódica ante la duda y el uso con criterio clínico de las imágenes y laboratorio.

\section{Referencias}

1.- Kwok M, Kim M, Gorelick M: Evidence-Based Approach to the Diagnosis of Appendicitis in Children. Pediatric Emergency Care 2004; 20: 690-701.

2.- Golden R, Reginald H: Fitz, appendicitis, and the Osier connection A discursive review. Surgery 1995; 118: 504-9.

3.- Ellis H: The 100th birthday of apendicitis. BMJ 1986; 293: 20-7.

4.- Mirilas $P$, Skandalakis JE: Not just an appendix: Sir Frederick Treves, Arch Dis Child 2003; 88: 549-53.

5.- Williams G: Presidential Address: A History of Appendicitis, With Anecdotes Illustrating Its Importance. Ann Surg 1983; 197: 495-506.

6.- Kohan R: La apendicitis aguda en el niño (1). Revista Chilena de Pediatría 1932; 66-79.

7.- Díaz Bordeu E: Diagnóstico diferencial de la Apendicitis Aguda. Rev Chil Pediatr 1948; 19: 208-11.

8.- Sakellaris G, Tilemis $S$, Charissis G: Acute appendicitis in preschool-age children. Eur J Pediatr 2005; 164: 803.

9.- Nance M, Adamson W, Hedrick H: Appendicitis in the young child: A continuing diagnostic challenge. Pediatr Emerg Care 2000; 16: 160-2.

10.- Bachur R, Hennelly K, Callahan M, Chen C, Monuteaux $M$ : Diagnostic Imaging and Negative Appendectomy Rates in Children: Effects of Age and Gender. Pediatrics 2012; 129: 877-84.

11.- Brewster GS, Herbet ME: Medical myth: a digital rectal examination should be performed on all indivual with possible appendicitis. incidence and value of rectal examination in children with suspected appendicitis. West $\mathrm{J}$ Med 200; 173: 207-8.

12.- Dolz A, García R: Apendicitis Aguda en el niño. Rev Chil Pediatr 1976; 47: 121-5.

13.- Camacho E, Vera M: Apendicitis Aguda en la infancia: algunas consideraciones sobre 1821 enfermos operados en un Servicio de Urgencia Infantil. Rev Chil Pediatr 1961: 344-9.

14.- Vidal S, Toloza L, Cancino B: Evolución de la prevalencia de enteroparasitosis en la ciudad de Talca, Región del Maule, Chile. Rev Chil Infectol 2010; 27: 336-40.

15.- Zuñiga M: Programa Nacional de Control de la Tuberculosis, Manual de Organización y Normas Técnicas. Subsecretaria de salud Publica, División de Prevención y Control de Enfermedades. Gobierno de Chile, MINSAL, 2005.

16.- Toorenvliet B, Vellekoop A, Bakker R, et al: Clinical differentiation between acute appendicitis and acute mesenteric lymphadenitis in children. Eur J Pediatr Surg 2011; 21: 120-3.

17.- Buddingh KT, Wieselmann E, Heineman E, Broens PM: Constipation and Nonspecific Abdominal Pain in Teenage Girls Referred for Emergency Surgical Consultation. JPGN 2012; 54: 672-6.

18.- Espinoza J: Constipación en pediatría. Rev Chil Nutr 2002; 29 (3): 272-9.

19.- Vigil P, Riquelme R, Rivadeneira R, Aranda W: TeenSTAR: Una opción de madurez y libertad. Programa de educación integral de la sexualidad, orientado a adolescentes. Rev Med Chile 2005; 133: 1173-82.

20.- MINSAL, DEIS, Estadísticas de Natalidad y Mortalidad Chile, Año 2009. deis.minsal.cl/vitales/anuarios/2009/ Anuario_2009_vitales.pdf

21.- Schonhaut L, Millan T: La educación sexual debe comenzar en el hogar y continuar en la escuela. Rev Chil Pediatr 2011; 82: 454-60.

22.- Morrow E, Newman K: Current management of apendicitis. Sem Ped Surg 2007; 16: 34-40.

23.- Jaffe B, Berger D: The Appendix. En: Brunicardi F., Andersen D., Billiar T. ed. Schwatrz's Principles of Surgery, Unite States of America, $9^{\circ}$ Edicion. Editorial The McGraw-Hill Companies, Inc. 2010; Capítulo 30.

24.- Livingston EH, Woodward WA, Sarosi GA: Disconnect between incidence of nonperforated and perforated appendicitis: implications for pathophysiology and management. Ann Surg 2007; 245: 886-92.

25.- Artigas R: La penicilina en paracaidas. En: Artigas R. ed. Sucesos en la vida de un cirujano, Santiago: Editorial Universitaria, 1989; 31.

26.- Keyzer C, Zalcman M, De Maertelaer V, et al: Comparison of US and unenhanced multi-detector row $\mathrm{CT}$ in patients suspected of having acute appendicitis. Radiology 2005; 236: 527-34.

27.- Ang A, Chong NK, Daneman A: Pediatric appendicitis in "real-time": The value of sonography in diagnosis and treatment. Pediatr Emerg Care 2001; 17: 334-40.

28.- Martin A, Vollman D, Adler B, Caniano DA: CT Scans May Not Reduce the Negative Appendectomy Rate in Children. J Ped Surg 2004; 39: 886-90.

29.- Bennett J, Boddy A, Rhodes M: Choice of Approach 
for Appendicectomy: A Meta-analysis of Open versus Laparoscopic Appendicectomy. Surg Laparosc Endosc Percutan Tech 2007; 17: 245-55.

30.- Liu Z, Zhang P, Ma Y: Laparoscopy or not: A Metaanalysis of the Surgical Effects of Laparoscopic versus Open Appendicectomy. Surg Laparosc Endosc Percutan Tech 2010; 20: 362-70.

31.- Sauerland S, Jaschinski T, Neugebauer EAM: Laparoscopic versus open surgery for suspected apendicitis (Review). The Cochrane Library 2010, Issue 10.

32.- Khan M, Vidya AE, Lee R: Are routine peritoneal fluid cultures during appendicectomy justified? Ir J Med Sci 2007; 176: 37-40.

33.- Guillet-Carubaa C, Cheikhelardb A, Guilleta M: Bacteriologic epidemiology and empirical treatment of pediatric complicated apendicitis. Diagnostic Microbiology and Infectious Disease 2011; 69: 376-81.
34.- Chan K, Lee K, Mou J, Cheung S, Sihoe J, Tam Y: Evidence-based adjustment of antibiotic in pediatric complicated appendicitis in the era of antibiotic resistance. Pediatr Surg Int 2010; 26: 157-60.

35.- Snelling C, Poenaru D, Drover J: Minimum postoperative antibiotic duration in advanced appendicitis in children: a review. Pediatr Surg Int 2004; 20: 838-45.

36.- Taylor M, Emil S, Nguyen N, Ndiforchu F: Emergent vs urgent appendectomy in children: a study of outcomes. J Ped Surg 2005; 40 (12): 1912-5.

37.- Lam M, Singh A, Kaewlai R, Novelline R: Magnetic Resonance of Acute Appendicitis: Pearls and Pitfalls. Curr Probl Diagn Radiol 2008; 37: 57-66.

38.- Muenzer J, Jaffe D, Schwulst S, et al: Evidence for a Novel Blood RNA Diagnostic for Pediatric Appendicitis The Riboleukogram. Pediatr Emer Care 2010; 26: 333 8. 